\title{
Radio Research in Australia
}

$\mathrm{T}$ HE Radio Research Board, which was set up some years ago under the Commonwealth of Australia Council for Scientific and Industrial Research, continues to carry out considerable research into several of the fundamental problems of radio communication. The results of some of this work have recently been described in a series of ten scientific papers published in Melbourne in three reports of the Radio Research Board. A foreword to these reports states that the co-operation of several bodies in Australia has greatly assisted the work of the Board. In particular, the Postmaster General's Department contributed half the cost of the investigations during the financial year 1928-29, and threequarters of this cost in the succeeding years. The Universities of Sydney and Melbourne have provided the necessary laboratory facilities for the location of the staff conducting the work, while the Department of Defence has also assisted with the loan of apparatus and in other ways. It is to be noted that several of the authors of the papers received their early research training in Great Britain with the Radio Research Board organisation under the Department of Scientific and Industrial Research.

The first report of the series under review contains four papers", of which one "On the Rotation of the Plane of Polarisation of Long Radio Waves" has been previously published in the Proceedings of the Royal Society, 1934. The other three have also been published previously in the Journal of the Institution of Engineers, Australia. Two of these papers deal with the development of a radio field intensity measuring set for medium broadcast frequencies, and its use in the exploration of the field around some Australian broadcasting stations. As a result of this work data have been obtained on the effective conductivity of several types of ground, and the resulting attenuation accompanying the propagation of waves over this ground. At the same time, measurements were made on the intensities of indirect or downcoming waves at various distances, and on the general noise level due to atmospheric disturbances, with the view of determining the effective service range of certain broadcasting stations. The last paper in this series describes the development of a frequency recorder for the rapid and continuous measurement of audio frequencies, as required in an extension of the frequency-change method of determining the heights of reflecting layers in the ionosphere.

Report No. 7 contains a group of four papers describing investigations into the propagation of medium frequency electric waves in the ionosphere ${ }^{2}$. Each of these papers has already been published in Great Britain during 1934 or 1935 .

The last of the reports ${ }^{3}$ so far received contains two hitherto unpublished papers describing the study of atmospherics and their interference effect in radio reception in Australia. The first of these describes a series of observations of the directions and intensities of atmospheries carried out with two visual cathode ray direction-finders installed at the ends of a base line 570 miles long and operating on a wave-length of 3,000 metres. The sites selected for these instruments, at Canberra and Toowoomba respectively, were considered to be particularly suitable for obtaining data on sources of atmospherics in Queensland. With the aid of a short wave radio communication link, simultaneous visual observations were made over a period of four months, December 1933-March 1934. The results are illustrated in the paper by maps showing the distribution of the sources of atmospherics during this summer period, and also lines of equal mean intensity of disturbance produced by each source.

The location of these atmospherics sources shows a very satisfactory correlation with the reports of thunderstorms obtained from the ordinary meteorological records, and thus strongly confirms the previous deduction that the majority of, if not all, atmospherics in Australia originate in lightning flashes. An important inference made by the authors on this portion of the work is that for thunderstorm warnings for aircraft, the cathode ray directionfinder is much preferable to ordinary meteorological methods.

The shapes of the iso-intensity lines referred to above for daytime observations were found to be satisfactorily explained by the effect of the topography of the ground on the propagation of the electric disturbance constituting the atmospheric. After making allowance for this effect, both the day and night observations agree in indicating that all the sources were of the same mean intensity within reasonable limits ; in other words, the average power in a particular wave-band radiated by lightning flashes is sensibly constant. The distances at which the sources were located ranged up to about 2,500 miles from the observing stations.

The last paper in this series discusses the effect of atmospheric disturbances on broadcast reception conditions in Australia. The equivalent power of the radiation emitted by an average lightning flash is calculated, and the activity and distribution of lightning flashes in thunderstorms in various parts of Australia has been obtained from the meteorological records. With the aid of these data, the interference of atmospherics with the reception of broadcasting on different wave-lengths and at various field intensities has been calculated. Under the conditions considered in the paper, it is concluded that the proportional time of interference with the normal 4,000 hours of broadcasting per annum is very small. In the foreword to this report (No. 8) it is pointed out that some matters in this paper, such as the standards of reception assumed, may not be generally accepted by other experts on this subject.

${ }^{1}$ A. L. Green and G. Builder: "On the Rotation of the Plane of "Polarisation of Long Radio Waves"; A. L. Green and N. B. Wood : "A Field-Intensity Set"; G. H. Munro and A. L. Green : "MeasureAustralia at 200 Kilocycles per Second"; D. F. Martyn and H. B. Wood :"A Frequency Recorder". Radio Research Board, Report No, 6, Melbourne 1935.

i D. F. Martyn : "The Propagation of Medium Radio Waves in the Ionosphere" ; D. F. Martyn and A. L. Green: "The Characteristics of Downcoming Radio Waves"; V. A. Bailey and D. F. Martyn: "The Influence of Electric Waves on the Ionosphere" ; $\dot{D}$. $\dot{F}$. Martyn, R. O. Cherry and A. L. Green: "Long Distance Observations of Radio Waves of Medium Frequencies". Radio Research Board, Report No. 7, Melbourne 1935 .

3 G. H. Munro, H. C. Webster and A. J. Higgs: "Simultaneous Observations of Atmospherics with Cathode Ray Direction-Finders at with Reception". Radio Research Board, Report No. 8, Melbourne 1935 . 